\title{
Crecimiento de plantines de Prosopis alba en diferentes sustratos, contenedores y condiciones de vivero
}

\author{
Growth of Prosopis alba seedlings on different substrates, containers and nursery conditions
}

\author{
Carla S Salto a*, Leonel Harrand ${ }^{\text {a }}$, Gustavo Pedro Javier Oberschelp a, Mauricio Ewens ${ }^{\text {b }}$

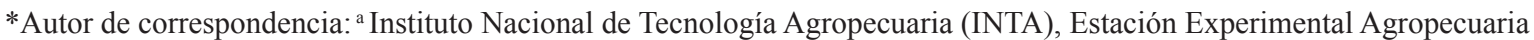 \\ Concordia, CC 34, CP 3200, Concordia, Entre Ríos, Argentina, salto.carla@inta.gob.ar

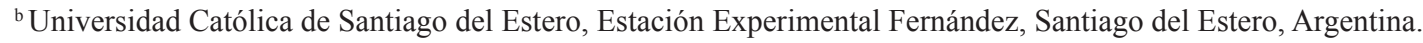

\begin{abstract}
SUMMARY
There is limited information about seedlings technology production of Prosopis alba ("algarrobo blanco" tree), a native species of commercial importance in Argentina. Therefore, the objective of this present work was to evaluate the effect of different substrates and containers on $P$. alba seedlings growth in two nurseries with contrasting environmental conditions. Composted pine bark and soil (CPT) and composted pine bark, perlite and vermiculite (CPV) mixtures were tested in 10 types of containers with different heights and volumes. For each combination of substrate and container, total porosity (PT), water retention (RA) and aeration porosity (PA) were measured. All these properties were influenced by container characteristics and substrates, showing the highest PT and PA values in CPV and bigger containers, while the RA was higher in CPT and smaller containers. Seedling growth, measured as seedling collar diameter (DAC) and total height (AT) was affected by the substrate and container type, showing higher growth in bigger containers. Furthermore, growth increased with volume and diameter of the container, being less influenced by the height. As a result, containers choice must be done based on the size and desired seedling production time, along with substrates that balance good porosity and water retention.
\end{abstract}

Key words: propagation; porosity; water retention; "algarrobo"; container size.

\section{RESUMEN}

Existe escasa información sobre las tecnologías de producción de plantines de algarrobo blanco (Prosopis alba), especie nativa de importancia comercial en Argentina. Por ello, el objetivo del trabajo fue evaluar el efecto de distintos sustratos y tipos de contenedores sobre el crecimiento de plantines de $P$. alba en dos viveros con condiciones ambientales contrastantes. Los sustratos probados fueron: corteza de pino compostada y tierra (CPT), y corteza de pino compostada, perlita y vermiculita (CPV). Se emplearon 10 tipos de contenedores con diferentes combinaciones de alturas y volúmenes, siendo las variables de análisis el diámetro al cuello (DAC) y altura total (AT) del plantín. Se determinaron sobre los contenedores las propiedades físicas de los sustratos: porosidad total (PT), porosidad de aireación (PA) y retención de agua (RA). Estas propiedades demostraron ser dependientes de las características del contenedor y del sustrato, registrándose los mayores valores de PT y PA en CPV y en contenedores de mayor volumen, mientras que la RA fue mayor en CPT y en contenedores de menor volumen. El crecimiento en DAC y AT fue afectado por el sustrato y el tipo de contenedor, presentándose los mayores incrementos en los contenedores de mayor capacidad. Se observó mayor crecimiento de los plantines con el aumento del volumen y diámetro del contenedor, siendo menor la influencia de la profundidad de los mismos. La elección del contenedor a utilizar debe realizarse en función del tamaño y momento del plantín deseado, con sustratos que equilibren una adecuada porosidad y retención de agua.

Palabras clave: propagación, porosidad, retención de agua, algarrobo, dimensiones de contenedores.

\section{INTRODUCCIÓN}

Prosopis alba Griseb. (algarrobo blanco) es una leguminosa arbórea de amplia distribución geográfica en Argentina, abarcando las regiones fitogeográficas del Parque Chaqueño y del Espinal (región centro-norte del país). Es una de las especies nativas de mayor valor comercial en el mercado de la madera aserrada, constituye un eje biológico estructural de sistemas productivos sustentables y contribuye a la recuperación de áreas degradadas; sus frutos son una importante fuente proveedora de materia prima para consumo humano y animal (López et al. 2001). Debido a sus cualidades este recurso forestal ha sido explotado de manera irracional provocando la degradación de sus bosques, por lo que se torna necesario emprender programas de forestación y enriquecimiento con esta valiosa especie.

La producción de plantines de calidad es un requisito indispensable para asegurar la supervivencia y el adecuado desarrollo de los bosques implantados (Salifu y Jacobs 2006). La elección del tipo de contenedor y la selección y 
manejo de los sustratos debe ocupar un lugar decisivo para obtener un plantín de calidad comercial, ya que el desconocimiento de la importancia de ello puede traer consecuencias económicas no deseadas al productor. Por esta razón, es importante contar con conocimientos y tecnologías de producción comercial de algarrobo blanco, aspecto sobre el que existe escasa información.

La combinación y preparación de los componentes en la formulación de un sustrato deben ser cuidadosamente estudiadas según los requerimientos de cada especie, pues el volumen restringido de los contenedores exige óptimas propiedades físicas (porosidad total, porosidad de retención de agua, entre otras) y químicas (como $\mathrm{pH}$ y conductividad eléctrica) para el crecimiento, es decir, que el sustrato proporcione soporte físico, provea agua, permita el intercambio gaseoso y sea reservorio de nutrientes dentro del contenedor (Landis 1990).

Existe una gran heterogeneidad de los sustratos utilizados en la producción de plantines forestales, dado que la mayoría de los viveristas formulan sus propios sustratos con materiales disponibles localmente. La corteza de pino (Pinus elliottii Engelm. y/o P. taeda L.) con diferentes grados de descomposición es una de las más utilizadas en la Mesopotamia Argentina (provincias de Misiones, Corrientes y Entre Ríos), variando considerablemente en sus propiedades físicas y químicas debido a los diferentes niveles de degradación y tamaño de sus partículas. Asimismo, es común el empleo de otros materiales como suelo, turbas subtropicales, turba de musgo Sphagnum spp., perlita y vermiculita (García et al. 2005).

En general, en la producción de plantines de algarrobo blanco se utilizan envases de polietileno como contenedor y mezclas constituidas por mantillo de monte y tierra común, o por tierra y arena como sustrato (Salto et al. 2013). Algunas investigaciones indican que el aumento de la proporción de suelo en la composición de un sustrato disminuye los costos pero aumenta el peso de los contenedores (García et al. 2005, Cunha et al. 2005), genera condiciones de anoxia por encharcamiento y dificulta la extracción del plantín del contenedor (Salto et al. 2013). Por otro lado, la extracción continua de la "tierra de monte" provoca un efecto ambiental negativo aumentando la erosión de los suelos, haciendo necesario el desarrollo de materiales alternativos como sustratos para reducir o eliminar el uso de tierra en la producción de plantines (Acosta Duran et al. 2008).

La ventaja que presenta la producción de plantas en contenedor radica en la facilidad de su manejo en vivero, la menor exposición a daños mecánicos y un mejor control de la nutrición (Gomes et al. 2003, Mathers et al. 2007). Sin embargo, la mayor limitación es el confinamiento que sufren las raíces, por lo que resulta importante priorizar metodologías de producción que no causen deformaciones en las mismas (Brachtvogel y Malavasi 2010).

En ese sentido, el volumen, altura y diámetro del contenedor varía en función de las especies que se quieren propagar y del tiempo de permanencia en el vivero (Nicoloso et al. 2000), además de ejercer influencia directa sobre la cantidad de sustrato a ser empleado, el espacio que se ocupará en el vivero, la mano de obra, la cantidad de insumos utilizados, la calidad y el costo final del plantín forestal (Gomes et al. 2003). En Argentina, el mercado ofrece contenedores de diferentes tamaños y formas, sin información de su aptitud para la producción de especies forestales nativas (Salto et al. 2013).

Las investigaciones orientadas a la caracterización física y química de los materiales empleados en la elaboración de sustratos para la producción de plantas forestales en contenedor, la respuesta de las plantas a estos medios de crecimiento $y / o$ a las dimensiones de los contenedores, son incipientes y escasas en la Argentina.

Dentro de este contexto, y ante la necesidad de generar información sobre la producción de plantas de P. alba, se plantea como hipótesis que el crecimiento en diámetro al cuello y altura total y el tiempo de formación del cepellón en plantines de algarrobo blanco se encuentran directamente relacionados con el tamaño del contenedor, las propiedades físicas de los sustratos y las condiciones ambientales de los viveros donde se producirán los mismos. Por ello, los objetivos del trabajo son caracterizar mediante propiedades físicas dos mezclas de sustrato en combinación con diez tipos de contenedores y evaluar el efecto de estas propiedades sobre el crecimiento en plantines de $P$. alba en dos viveros con condiciones ambientales contrastantes (Parque Chaqueño y Espinal).

\section{MÉTODOS}

Los ensayos se realizaron en dos viveros de las provincias de Entre Ríos y Santiago del Estero, Argentina. El vivero forestal de la Estación Experimental Agropecuaria Concordia, perteneciente al Instituto Nacional de Tecnología Agropecuaria (Concordia, Entre Ríos), está ubicado a $31^{\circ} 22^{\prime} \mathrm{S}$ y $58^{\circ} 07^{\prime} \mathrm{O}$ con una altitud de $47 \mathrm{~m}$ s.n.m., es una región caracterizada como templado-cálida sin estación seca, con temperatura media anual de $18,7^{\circ} \mathrm{C}$, precipitación anual promedio de $1.345 \mathrm{~mm}$, con valores máximos y mínimos de $2.193 \mathrm{~mm}$ y $868 \mathrm{~mm}$, respectivamente. Durante el periodo de ensayo se registró una temperatura media de $23,6^{\circ} \mathrm{C}$, máxima media de $30,3{ }^{\circ} \mathrm{C}$ (valores diarios entre $20,3^{\circ} \mathrm{C}$ y $41,4^{\circ} \mathrm{C}$ ) y mínima media de $17,5^{\circ} \mathrm{C}$ (valores diarios entre $9,0^{\circ} \mathrm{C}$ y $28^{\circ} \mathrm{C}$ ). El vivero forestal de la Estación Experimental Fernández de la Universidad Católica de Santiago del Estero (Fernández, Santiago del Estero) está ubicado a $27^{\circ} 55^{\prime} \mathrm{S}$ y $63^{\circ} 55^{\prime} \mathrm{O}$ a una altitud de $160 \mathrm{~m}$ s.n.m., presenta un régimen monzónico con precipitaciones que varían entre 500 y $550 \mathrm{~mm}$ anuales de noviembre a marzo y una temperatura media anual de $20^{\circ} \mathrm{C}$. En el transcurso del ensayo se registró una temperatura media de $27,3{ }^{\circ} \mathrm{C}$, máxima media de $34,5{ }^{\circ} \mathrm{C}$ (valores diarios entre $24,0{ }^{\circ} \mathrm{C}$ y $41,8^{\circ} \mathrm{C}$ ) y mínima media de $20,3{ }^{\circ} \mathrm{C}$ (valores diarios entre $12,5^{\circ} \mathrm{C}$ y $\left.27,2^{\circ} \mathrm{C}\right)$. 
Se emplearon semillas de árboles semilleros de $P$. alba provenientes del Parque Chaqueño. El tratamiento pre-germinativo utilizado consistió en la inmersión de las semillas en agua a $100^{\circ} \mathrm{C}$, manteniéndolas sumergidas hasta alcanzar temperatura ambiente, durante $24 \mathrm{~h}$. La siembra se realizó directamente sobre los contenedores durante la primera semana de octubre 2013 en Concordia y en la segunda semana de noviembre de 2013 en Fernández. En Concordia el riego aplicado fue por microaspersores bajo un sistema automatizado con tres riegos diarios de 45 minutos cada uno, mientras que en Fernández el riego se realizó en forma manual con manguera y a baja presión hasta saturación tres veces al día. El plan de fertilización empleado fue el indicado por Silveira et al. (2001) para cubrir los requerimientos nutricionales de plantines de Eucalyptus grandis, efectuándose el mismo dos veces por semana a partir de los 30 días posteriores a la siembra hasta la culminación del ensayo. Los sustratos empleados fueron mezclas de: i) corteza de pino compostada y tierra en una proporción $3: 1 \mathrm{v} / \mathrm{v}$ y ii) corteza de pino compostada, perlita (Perlome ${ }^{\circledR}$ Grueso) y vermiculita (Intersum ${ }^{\circledR}$ Mediano F1) en proporción 2:1:1 v/v. Se evaluaron diez tipos de contenedores de Dassplastic del Mercosur ${ }^{\mathbb{B}}$ (cuadro 1). El diseño experimental fue de parcelas divididas en bloques completos al azar con cinco repeticiones y parcelas de 36 plantas. Las variables de análisis fueron diámetro al cuello y altura total del plantín medidos a los $60,90,120 \mathrm{y}$, en Concordia, una medición extra a los 150 días, empleando calibre digital y regla milimetrada. Luego de cada medición se realizaron muestreos para seguimiento del proceso de formación de raíces y del cepellón en cada uno de los tiempos. Para el análisis se empleó un modelo lineal mixto para parcelas divididas con mediciones repetidas en el tiempo.
En laboratorio se midieron en los sustratos la conductividad eléctrica y el $\mathrm{pH}$ por el método de dilución 1:2 (Torres et al. 2010), empleando un equipo HANNA ${ }^{\circledR}$ HI4522. Se efectuó la determinación de las propiedades físicas de porosidad total, porosidad de aireación y porosidad de retención de agua de los sustratos en los contenedores evaluados de acuerdo a la metodología propuesta por Landis (1990). Para el sustrato corteza de pino compostada y tierra, solo se realizaron las determinaciones de estas propiedades en los contenedores con la mezcla de Concordia, por limitaciones en la disponibilidad de muestras procedentes de Fernández. Si bien cada vivero empleó tierra del lugar para las mezclas, estas se consideraron similares y se asumió a la mezcla CPT de ambos viveros como un mismo sustrato. Se empleó un diseño completamente aleatorizado con arreglo factorial de tratamientos, con diez repeticiones por contenedor.

Los análisis estadísticos se realizaron con el programa SAS v.9.1 (SAS Institute Inc. 2009) con el procedimiento MIXED. Para el caso de datos con mediciones repetidas se empleó además la instrucción REPEATED para la especificación de la estructura de covarianza. Cuando se obtuvieron diferencias significativas entre los factores $(P \leq 0,05)$, se realizaron pruebas de comparación de medias mediante la sentencia LSMEANS/SLICE opción ADJUST=Tukey, generando una comparación conjunta de los niveles de los demás factores en cada uno de los niveles del factor indicado (Correa Londoño 2004). En función de los resultados obtenidos en las variables morfológicas, se consideraron los efectos simples del factor sustrato y todas las combinaciones de tratamientos (sustrato x contenedor) en los diferentes tiempos de medición.

Cuadro 1. Características de los contenedores evaluados.

Containers characteristics.

\begin{tabular}{cccccccc}
\hline Modelo & $\begin{array}{c}\text { Volumen } \\
\left(\mathrm{cm}^{3}\right)\end{array}$ & $\begin{array}{c}\text { Diámetro } \\
\text { superior }(\mathrm{mm})\end{array}$ & $\begin{array}{c}\text { Diámetro } \\
\text { inferior }(\mathrm{mm})\end{array}$ & $\begin{array}{c}\text { Medida } \\
\text { superior }(\mathrm{mm})\end{array}$ & $\begin{array}{c}\text { Altura } \\
\text { contenedor }(\mathrm{mm})\end{array}$ & $\begin{array}{c}\text { Densidad de plantas } \\
\left(\text { plantas } \mathrm{m}^{-2}\right)\end{array}$ & Forma $^{*}$ \\
\hline R72 & 72 & 37 & 20 & - & 90 & 423 & TC \\
C90 & 90 & - & - & $38 \times 38$ & 96 & 423 & TP \\
R100 & 100 & 35 & 15 & - & 140 & 532 & TC \\
R110 & 110 & 35 & 6 & - & 180 & 532 & TC \\
R125 & 125 & 40 & 10 & - & 150 & 358 & TC \\
C125 & 125 & - & - & $42 \times 42$ & 117 & 318 & TP \\
R140 & 140 & 40 & 18 & - & 150 & 358 & TC \\
C140 & 140 & - & - & $37 \times 37$ & 142 & 424 & TP \\
R250 & 250 & 64 & 43 & - & 137 & 204 & TC \\
R270 & 270 & 50 & 41 & - & 180 & 209 & TC \\
\hline
\end{tabular}

* TC: tronco cónica; TP: tronco piramidal de base cuadrada. 


\section{RESULTADOS}

El sustrato formulado con corteza de pino compostada y tierra (mezcla obtenida con tierra de cada lugar de ensayo), fue ligeramente más ácido ( $\mathrm{pH} 5,1)$ que el sustrato de corteza de pino compostada, perlita y vermiculita $(\mathrm{pH} 6,2)$. Los valores de conductividad eléctrica fueron más bajos en el sustrato con presencia de perlita y vermiculita $(208 \mu \mathrm{S}$ $\mathrm{cm}^{-1}$ ) comparados con los valores obtenidos en el sustrato de corteza de pino compostada y tierra (332 y $542 \mu \mathrm{S} \mathrm{cm}^{-1}$ para Concordia y Fernández, respectivamente).

Se detectaron interacciones significativas entre los contenedores y los sustratos ensayados para las propiedades físicas (cuadro 2).

Cuadro 2. Análisis de la varianza para las relaciones de porosidades: porosidad total (PT), porosidad de aireación (PA) y retención de agua (RA) en los sustratos evaluados.

Analysis of variance for relations of porosities: total porosity (PT), aeration porosity (PA) and water retention (RA) of the substrates evaluated.

\begin{tabular}{lcccc}
\hline \multicolumn{1}{c}{ FV } & GL & \multicolumn{3}{c}{ Propiedades físicas de los sustratos } \\
& & PT & PA & RA \\
\hline $\begin{array}{l}\text { Contenedor } \\
\text { Sustrato }\end{array}$ & 9 & $<0,0001^{* * *}$ & $<0,0001^{* * *}$ & $<0,0001^{* * *}$ \\
$\begin{array}{l}\text { Contenedor } \\
\text { x Sustrato }\end{array}$ & 9 & $<0,0001^{* * *}$ & $<0,0001^{* * *}$ & $<0,0001^{* * *}$ \\
\hline
\end{tabular}

$*=P<0,05 ; * *=P<0,01 ; * * *=P<0,001$.
En el sustrato formulado con corteza de pino compostada, perlita y vermiculita se registraron los mayores valores de porosidad total y porosidad de aireación, mientras que en el sustrato de corteza de pino compostada y tierra, la retención de agua presentó los valores más altos (cuadro 3).

La porosidad total se presentó con valores similares entre todos los contenedores empleando el sustrato formulado con perlita y vermiculita, observándose el mayor valor en el contenedor R72 y el menor en el contenedor R110. Por otro lado, el mayor valor de porosidad total para el sustrato de corteza de pino compostada y tierra, se obtuvo en el contenedor $\mathrm{C} 125$ el cual se diferenció significativamente del resto de los contenedores (cuadro 3).

Los valores de porosidad de aireación se encontraron en un rango de $25-45 \%$ para todos los contenedores y sustratos. El mayor valor para esta propiedad se presentó en los contenedores $\mathrm{C} 125$ y R270 para los sustratos de corteza de pino compostada y tierra, y corteza de pino compostada, perlita y vermiculita, respectivamente (cuadro 3). En ambos sustratos la mayor retención de agua se obtuvo en el contenedor R72, y los menores valores se produjeron en los contenedores R110 y R270 (cuadro 3), contenedores que presentan los valores extremos de altura (cuadro 1).

Concerniente a ambas variables morfológicas, en Concordia la interacción entre todos los factores resultó significativa (cuadro 4). Para Fernández la interacción triple no fue estadísticamente significativa, implicando que las diferencias entre los tratamientos fueron consistentes a través del tiempo (cuadro 4).

Los mayores crecimientos promedios del diámetro al cuello, en todos los tiempos en Concordia, se obtuvieron

Cuadro 3. Porosidad total (PT), porosidad de aireación (PA) y retención de agua (RA) en los sustratos de corteza de pino compostada y tierra (CPT); corteza de pino compostada, perlita y vermiculita (CPV) en los diferentes contenedores.

Total porosity (PT), aeration porosity (PA) and porosity of water retention (RA) in composted pine bark and soil (CPT) and composted pine bark, perlite and vermiculite (CPV) mixtures in different containers.

\begin{tabular}{|c|c|c|c|c|c|c|}
\hline \multirow{3}{*}{$\begin{array}{l}\text { Contenedor } \\
\text { R72 }\end{array}$} & \multicolumn{3}{|c|}{$\mathrm{CPT}$} & \multicolumn{3}{|c|}{ CPV } \\
\hline & PT (\%) & PA (\%) & RA (\%) & PT (\%) & PA (\%) & RA $(\%)$ \\
\hline & $51,4 \quad b$ & $29,5 \mathrm{c}$ & 21,9 a & 58,7 a & $37,8 \quad \mathrm{~d}$ & $20,9 \quad a$ \\
\hline C90 & 47,5 de & $25,9 \mathrm{~d}$ & 21,6 a & $56,2 \mathrm{~cd}$ & $39,1 \mathrm{~cd}$ & 17,1 \\
\hline R100 & $51,2 \quad b$ & $35,8 \quad b$ & $15,4 \mathrm{~cd}$ & 56,6 bc & $42,7 \quad a b$ & 14,0 \\
\hline R110 & $50,6 \quad \mathrm{~b}$ & $36,1 \quad b$ & 14,6 cde & $54,5 \mathrm{e}$ & 43,3 a & $11,2 \mathrm{c}$ \\
\hline R125 & 49,6 bcd & $34,3 \quad b$ & $15,4 \mathrm{~cd}$ & 57,0 bc & 43,9 a & 13,2 c \\
\hline $\mathrm{C} 125$ & 59,6 a & $40,5 \quad a$ & $19,2 \quad b$ & $57,8 \quad a b$ & 40,3 & $17,5 \quad b$ \\
\hline R140 & 47,2 e & $31,3 \quad \mathrm{c}$ & $16,0 \quad \mathrm{c}$ & 55,2 de & $41,0 \quad b c$ & $14,2 \mathrm{c}$ \\
\hline C140 & $50,3 \quad b c$ & $36,3 \quad b$ & 14,0 de & 56,9 bc & 43,2 a & 13,7 c \\
\hline $\mathrm{R} 250$ & $50,2 \quad b c$ & $34,6 \quad b$ & $15,6 \mathrm{~cd}$ & 56,7 bc & $42,6 \quad a b$ & $14,1 \quad \mathrm{c}$ \\
\hline $\mathrm{R} 270$ & 48,2 cde & $35,2 \quad b$ & $13,0 \quad \mathrm{e}$ & $55,9 \mathrm{~cd}$ & $44,4 \quad \mathrm{a}$ & $11,5 \quad \mathrm{c}$ \\
\hline Media & 50,6 & 33,9 & 16,7 & 56,5 & 41,8 & 14,7 \\
\hline
\end{tabular}

Para cada columna, letras distintas indican diferencias significativas para la prueba de Tukey $(P \leq 0,05)$. 
Cuadro 4. Análisis de la varianza de medidas repetidas para diámetro al cuello (DAC) y altura total (AT) en algarrobo blanco para las dos condiciones de vivero evaluadas.

Analysis of variance for repeated measures of AT and DAC of Prosopis alba seedlings for the two conditions evaluated.

\begin{tabular}{|c|c|c|c|c|c|c|}
\hline \multirow[t]{2}{*}{ Fuente de variación } & \multirow[b]{2}{*}{ G.L. } & \multicolumn{2}{|c|}{ Concordia } & \multicolumn{3}{|c|}{ Fernández } \\
\hline & & $\mathrm{DAC}$ & AT & G.L. & $\mathrm{DAC}$ & AT \\
\hline Sustrato & 1 & $<0,0001^{* * *}$ & $<0,0001^{* * *}$ & 1 & $0,0163^{*}$ & $0,0900^{\mathrm{ns}}$ \\
\hline Contenedor & 9 & $<0,0001^{* * *}$ & $<0,0001^{* * *}$ & 9 & $<0,0001^{* * *}$ & $<0,0001^{* * *}$ \\
\hline Sustrato x Contenedor & 9 & $0,0034^{* *}$ & $0,0018^{* *}$ & 9 & $0,0167^{*}$ & $0,3994^{\mathrm{ns}}$ \\
\hline Tiempo & 3 & $<0,0001^{* * *}$ & $<0,0001^{* * *}$ & 2 & $<0,0001^{* * *}$ & $<0,0001^{* * *}$ \\
\hline Tiempo x Sustrato & 3 & $<0,0001^{* * *}$ & $<0,0001^{* * *}$ & 2 & $0,0133^{*}$ & $<0,0001^{* * *}$ \\
\hline Tiempo x Contenedor & 27 & $<0,0001^{* * *}$ & $<0,0001^{* * *}$ & 18 & $<0,0001^{* * *}$ & $<0,0001^{* * *}$ \\
\hline Tiempo x Contenedor x Sustrato & 27 & $0,0011^{* *}$ & $0,0086^{* *}$ & 18 & 0,8354 ns & $0,8272^{\mathrm{ns}}$ \\
\hline
\end{tabular}

$*=P<0,05 ; * *=P<0,01 ; * * *=P<0,001 ;$ ns: no significativo.

en el sustrato corteza de pino compostada, perlita y vermiculita $(P<0,0001)$, mientras que en Fernández fueron levemente mayores en corteza de pino compostada y tierra, y significativos a los 60 y 120 días $(P<0,01)$ (cuadro 5).

En Concordia a los 60 y 90 días los menores crecimientos en diámetro al cuello fueron registrados en el sustrato de corteza de pino compostada y tierra en los contenedores R110 y R250, y los mayores diámetros en los contenedores R72, C125 y R270 empleando el sustrato a base de corteza de pino compostada, perlita y vermiculita (cuadro 5). En las mediciones efectuadas a los 120 y 150 días, el crecimiento en diámetro de los plantines producidos en los diferentes contenedores, utilizando el sustrato a base de corteza de pino compostada y tierra, comparados con sus equivalentes en el sustrato de corteza de pino compostada, perlita y vermiculita, presentaron los menores crecimientos promedios. Las mayores diferencias se obtuvieron en los contenedores R250 y R270 empleando como sustrato corteza de pino compostada, perlita y vermiculita con respecto a los contenedores R100 y R110 con sustrato a base de corteza de pino compostada y tierra (cuadro 5). Cabe mencionar que los plantines producidos en el contenedor R270 con sustrato de corteza de pino compostada y tierra presentaron valores de diámetros similares a los obtenidos en el sustrato de corteza de pino compostada, perlita y vermiculita utilizando el contenedor R110 (cuadro 5).

En general, en Fernández se mantuvieron en el tiempo las diferencias detectadas en el diámetro al cuello, principalmente entre los 90 y 120 días. Los diámetros de los plantines producidos en los contenedores R250 y R270 empleando ambos tipos de sustratos fueron los mayores (cuadro 5).

En ambas localidades, independientemente del tipo de sustrato y el tiempo, los menores crecimientos en diámetro se presentaron en los contenedores R110 y R100.

Con respecto a la altura total en Concordia, los mayores crecimientos promedios se produjeron en el sustrato formulado con corteza de pino compostada, perlita y vermiculita (cuadro 6).A los 60 días, en los plantines producidos en los diez tipos de contenedores con el sustrato a base de corteza de pino compostada, perlita y vermiculita no se detectaron diferencias estadísticamente significativas, mientras que sí se detectaron diferencias significativas entre los contenedores en el sustrato a base de corteza de pino compostada y tierra (cuadro 6). Las mayores diferencias de crecimiento en altura a los 90, 120 y 150 días se produjeron en los contenedores R250 y R270 en el sustrato de corteza de pino compostada, perlita y vermiculita comparados con los contenedores R100, R110 empleando como sustrato la mezcla de corteza de pino compostada y tierra (cuadro 6).

En Fernández la interacción entre sustrato, contenedor y tiempo no resultó significativa, al igual que la interacción entre sustrato y contenedor. La falta de significancia del factor sustrato sugiere una mayor influencia del tipo de contenedor y del tiempo para el crecimiento en altura para las condiciones de vivero dadas (cuadro 4). Las alturas totales medias para los sustratos pueden ser consideradas diferentes $(P<0,05)$ solamente a los 60 días posteriores a la siembra, obteniéndose en este caso las mayores alturas promedio en el sustrato formulado con corteza de pino compostada y tierra, presentándose luego crecimientos similares de altura en ambos sustratos. En todos los tiempos e independientemente del sustrato, los mayores crecimientos en altura se produjeron en los contenedores de mayores dimensiones (R250 y R270), mostrando diferencias altamente significativas con respecto a los contenedores de menores dimensiones (R100, R110 y R72) (cuadro 7).

El tiempo de formación del cepellón estuvo en función del tamaño del contenedor siendo más rápida en contenedores de menor capacidad. Se observó mejor desarrollo y abundancia de raicillas secundarias en el sustrato de corteza de pino compostada, perlita y vermiculita, presentando el cepellón una consistencia firme y sin disgregación al 
Cuadro 6. Altura total media $(\mathrm{cm})$ en algarrobo blanco en diferentes contenedores y sustratos (CPT: sustrato corteza de pino compostada y tierra; CPV: sustrato corteza de pino compostada, perlita y vermiculita) evaluados para Concordia.

Total height (AT) of Prosopis alba in different treatments (CPT: composted pine bark and soil; CPV: composted pine bark, perlite and vermiculite) evaluated in Concordia.

\begin{tabular}{|c|c|c|c|c|c|c|c|c|}
\hline Sustrato & Contenedor & $60 c$ & días & $90 \mathrm{~d}$ & lías & 120 días & & 0 días \\
\hline $\mathrm{CPV}$ & $\mathrm{R} 250$ & 9,1 & $\mathrm{a}$ & 18,1 & $\mathrm{ab}$ & $31,7 \quad a b$ & 38,8 & $\mathrm{a}$ \\
\hline CPV & $\mathrm{R} 270$ & 9,0 & $\mathrm{a}$ & 19,6 & $\mathrm{a}$ & $32,0 \quad \mathrm{a}$ & 36,6 & $a b$ \\
\hline $\mathrm{CPV}$ & $\mathrm{C} 125$ & 8,8 & $\mathrm{a}$ & 17,0 & bcd & 28,4 abc & 33,4 & $a b c$ \\
\hline $\mathrm{CPV}$ & C90 & 9,0 & $\mathrm{a}$ & 16,6 & bcde & 28,3 abcd & 32,9 & bcd \\
\hline $\mathrm{CPV}$ & R125 & 9,3 & $\mathrm{a}$ & 17,4 & $a b c$ & 28,4 abc & 32,7 & bcd \\
\hline $\mathrm{CPT}$ & $\mathrm{R} 270$ & 6,9 & bcd & 15,4 & cde & $27,3 \mathrm{bcd}$ & 31,7 & bcde \\
\hline CPV & R140 & 9,1 & $\mathrm{a}$ & 16,4 & bcde & 26,7 cde & 31,1 & bcdef \\
\hline $\mathrm{CPV}$ & $\mathrm{C} 140$ & 8,5 & $\mathrm{a}$ & 16,5 & bcde & 25,7 cdef & 30,7 & cdefg \\
\hline $\mathrm{CPV}$ & R110 & 8,9 & $\mathrm{a}$ & 16,6 & bcde & 25,7 cdef & 30,3 & cdefg \\
\hline $\mathrm{CPV}$ & R72 & 8,9 & $\mathrm{a}$ & 15,3 & cdef & 24,3 cdefgh & 28,5 & cdefgh \\
\hline $\mathrm{CPT}$ & R125 & 7,2 & $\mathrm{~b}$ & 14,7 & defg & 25,4 cdef & 28,3 & cdefgh \\
\hline CPV & R100 & 8,6 & $\mathrm{a}$ & 15,7 & bcde & 24,2 defgh & 28,2 & cdefgh \\
\hline $\mathrm{CPT}$ & $\mathrm{R} 140$ & 6,9 & $\mathrm{bc}$ & 14,4 & efgh & 24,4 cdefg & 27,5 & defgh \\
\hline CPT & $\mathrm{C} 125$ & 6,2 & bcd & 12,4 & ghi & 22,6 efghi & 26,7 & efghi \\
\hline CPT & $\mathrm{C} 140$ & 6,3 & bed & 12,8 & fghi & 22,0 fghi & 25,8 & fghi \\
\hline CPT & $\mathrm{R} 250$ & 5,9 & $\mathrm{~d}$ & 11,7 & $\mathrm{i}$ & 21,8 fghi & 25,5 & fghi \\
\hline $\mathrm{CPT}$ & C90 & 6,3 & bcd & 11,8 & $\mathrm{i}$ & 21,0 ghi & 24,6 & ghi \\
\hline CPT & R72 & 6,4 & bcd & 12,0 & hi & 21,0 ghi & 23,7 & \\
\hline CPT & R110 & 6,5 & bcd & 11,7 & $\mathrm{i}$ & 20,1 hi & 23,0 & \\
\hline $\mathrm{CPT}$ & R100 & 6,2 & $\mathrm{~cd}$ & 10,8 & $\mathrm{i}$ & $18,7 \quad \mathrm{i}$ & 21,4 & \\
\hline \multicolumn{2}{|l|}{ CPV media } & 8,9 & A & 16,9 & A & $27,5 \mathrm{~A}$ & 32,3 & \\
\hline \multicolumn{2}{|l|}{ CPT media } & 6,5 & $\mathrm{~B}$ & 12,8 & B & $22,4 \mathrm{~B}$ & 25,8 & \\
\hline
\end{tabular}

Para cada columna, letras distintas indican diferencias significativas para la prueba de Tukey $(P \leq 0,05)$.

Cuadro 7. Altura total media $(\mathrm{cm})$ en algarrobo blanco en diferentes contenedores y sustratos (CPT: sustrato corteza de pino compostada y tierra; CPV: sustrato corteza de pino compostada, perlita y vermiculita) evaluados para Fernández.

Total height (AT) of Prosopis alba in different treatments (CPT: composted pine bark and soil; CPV: composted pine bark, perlite and vermiculite) evaluated in Fernández.

\begin{tabular}{ccccc}
\hline Factores & 60 días & 90 días & 120 días \\
\hline R270 & 32,2 a & 44,3 a & 48,5 a \\
R250 & 30,0 ab & 42,3 ab & 46,0 ab \\
C140 & 28,9 bc & 38,7 bc & 41,9 bc \\
R140 & 27,2 cd & 36,9 cd & 39,8 cd \\
C125 & 27,5 bcd & 36,9 cd & 38,9 cd \\
R125 & 25,9 d & 34,9 de & 37,3 de \\
C90 & 26,3 cd & 34,4 def & 35,9 de \\
R110 & 23,0 e & 31,9 ef & 34,5 e \\
R72 & 25,0 de & 32,3 ef & 34,0 e \\
R100 & 22,7 e & 30,8 f & 33,2 e \\
CPT & 28,6 A & 37,0 A & 39,9 A \\
CPV & 25,2 B & 35,8 A & 38,1 A \\
\hline
\end{tabular}

Para cada columna, letras distintas indican diferencias significativas para la prueba de Tukey $(P \leq 0,05)$. 

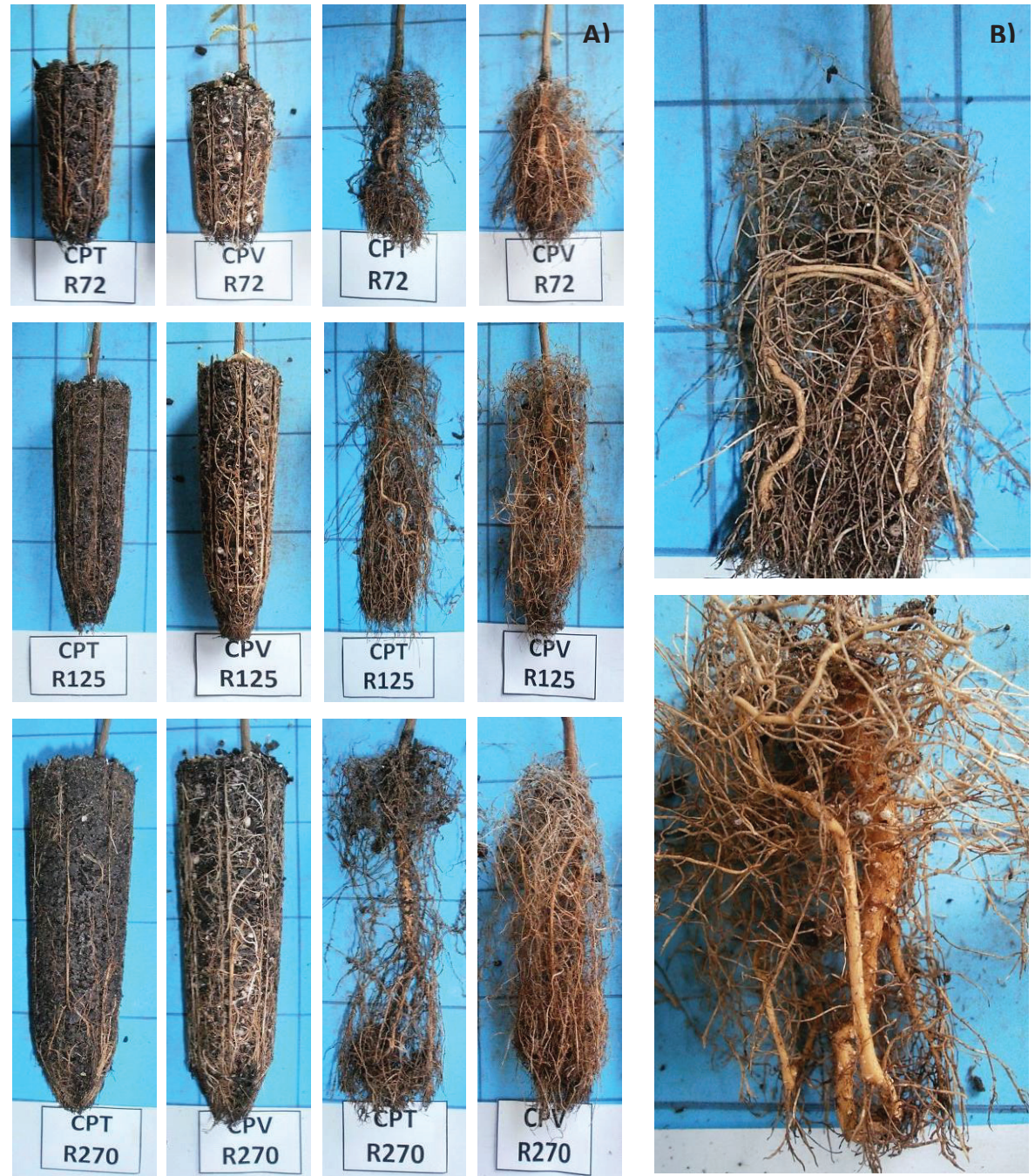

Figura 1. A) Cepellones de algarrobo blanco obtenidos en diferentes tamaños de contenedor en los dos sustratos ensayados (CPT: sustrato corteza de pino compostada y tierra; CPV: sustrato corteza de pino compostada, perlita y vermiculita) a los 120 días de edad (cuadros de referencia en foto de 5 x $5 \mathrm{~cm}$ ). B) Deformaciones radicales.

A) Root balls from Prosopis alba seedlings obtained with different container sizes and substrates at 120 days (reference frame picture of 5 $x 5 \mathrm{~cm}$ ). B) Root deformations.

ser extraído del contenedor (figura 1A). En algunas de las plantas muestreadas se encontraron problemas de deformaciones de la raíz principal en los contenedores de forma cuadrada y de pequeña capacidad, en ambos sustratos (figura 1B).

\section{DISCUSIÓN}

El pH y conductividad eléctrica del sustrato tienen una marcada influencia en el crecimiento radicular de las plantas producidas en contenedor y es importante conocerlos para seleccionar un sustrato adecuado y programar las actividades culturales (Mathers et al. 2007). Aunque las especies forestales son capaces de tolerar un intervalo relativamente amplio de $\mathrm{pH}$, es recomendable mantenerlo entre 5,4 y 6,8 para asegurar la disponibilidad de nutrientes y evitar enfermedades (Landis 1990). El valor de $\mathrm{pH}$ en el sustrato de corteza de pino compostada y tierra es ligeramente más ácido que lo recomendado por los autores anteriormente mencionados. Esto se debe posiblemente a que este sustrato contiene una mayor proporción de corteza de pino compostada (75\%) que el sustrato de corteza de pino compostada, perlita y vermiculita (50\%), o bien, a la presencia de vermiculita en este último, reconocida por estabilizar el $\mathrm{pH}$ de las mezclas en valores cercanos a la neutralidad (Indrasumunar y Gresshoff 2013). Los valores de conductividad eléctrica se encuentran dentro del rango calificado como bajo a muy bajo, siendo el rango normal para el método de medición empleado, valores entre 760 y $1.250 \mu \mathrm{S} \mathrm{cm}^{-1}$ (Torres et al. 2010). 
Según los resultados obtenidos, se hace evidente que las propiedades físicas evaluadas dependen de las características del contenedor (dimensiones y forma) y del tipo de sustrato que se esté empleando. Los valores de porosidad total y porosidad de aireación en el sustrato de corteza de pino compostada, perlita y vermiculita son semejantes a los encontrados por Salto et al. (2013), que indican que la presencia de corteza de pino y perlita en la mezcla favorece la capacidad de aireación y el drenaje.

La porosidad total es una medida de la capacidad que tiene un sustrato de retener aire y agua pero no brinda indicaciones del tamaño de los poros (macro y microporos), los cuales determinan la tasa de drenaje e intercambio gaseoso (Landis 1990, Lemaire et al. 2005). Para la producción de plantines de especies leñosas en contenedores se recomiendan valores mayores a $50 \%$ de porosidad total (Mathers $e t$ al. 2007), umbral no alcanzado para la mezcla de corteza de pino compostada y tierra en algunos contenedores.

Landis (1990) y Mathers et al. (2007) indican rangos óptimos de 20-30\% de porosidad de aireación pero señalan que los valores de esta propiedad varían de acuerdo a las características de los distintos componentes de los sustratos, el grado de compactación y a los métodos de medición de sus propiedades. En este estudio el límite superior de la porosidad de aireación se encuentra por encima del valor recomendado (45\%). En los dos sustratos evaluados, los contenedores R72 y C90 presentaron los menores valores de porosidad de aireación. Estos contenedores se caracterizan por presentar el menor volumen y la menor altura, siendo esta última característica, la que afecta negativamente a la porosidad de aireación (Mathers et al. 2007). Los sustratos mal aireados provocan que las raíces se agrupen en la parte superior del contenedor. Esto se traduce en un débil enraizamiento, ya que las raíces apenas exploran la parte central del sustrato y tienden a enrollarse afectando la supervivencia de la planta (Masaguer y López Cuadrado 2006). Por otro lado, una proporción excesiva de macroporos provoca una mayor aireación y una menor eficiencia en el uso del agua y fertilizantes (Mathers et al. 2007).

Los valores de retención de agua pueden considerase ligeramente menores a los niveles recomendados (Mathers et al. 2007). El mayor valor de esta propiedad para la mezcla de corteza de pino compostada y tierra, se debe a que esta formulación cuenta con una proporción de tierra, que se traduce en una mayor cantidad de microporos y en consecuencia, el medio puede saturarse, encharcarse y presentar problemas de aireación y drenaje. La retención de agua, a su vez, se encuentra relacionada al concepto de capacidad de contenedor, que se define como el porcentaje volumétrico de agua que es retenido por el sustrato en un contenedor de altura $\mathrm{h}$, una vez saturada y dejado drenar libremente sin sufrir evaporación (Mascarini et al. 2012). Para contenedores con diferentes alturas, la capacidad de contenedor será menor cuanto mayor sea la altura, por lo tanto la correcta elección del sustrato está directamente relacionada a la altura del contenedor (Vence et al. 2013).
Estudios realizados por Salto et al. (2013) indican que los crecimientos en diámetro y altura de plantines de algarrobo blanco y negro son favorecidos en sustrato de corteza de pino compostada, perlita y vermiculita en proporción 2:1:1 v/v. Díaz (2009) no recomienda el empleo de la corteza de pino compostada como sustrato en proporciones iguales o superiores al $50 \%$ para la producción de plantines de algarrobo blanco pues el crecimiento en diámetro y altura se ve restringido. Los resultados obtenidos en este estudio no concuerdan con lo indicado por Díaz (2009), ya que no se detectaron problemas de crecimiento en diámetro o altura de los plantines por el empleo de la corteza de pino compostada como parte componente del sustrato, lo cual puede atribuirse a un efecto regulador conjunto de la fertilización y/o de la vermiculita empleada en este trabajo, evitando valores de $\mathrm{pH}$ extremadamente bajos, comunes en estos tipos de sustratos.

Lemaire et al. (2005) indican que las dimensiones del contenedor, fundamentalmente la sección transversal, tienen una marcada influencia en el crecimiento de los plantines y en la calidad del cepellón. Al aumentar la sección del contenedor, los diámetros y alturas que se obtienen son mayores comparados con contenedores de sección más estrecha, pues se presenta una mayor capacidad de captación de agua de riego y la densidad de raicillas del cepellón pueden extenderse más y mejor en los contenedores de mayor sección. Tanto para altura total como para diámetro al cuello, los contenedores R250 y R270 presentaron diferencias estadísticas significativas en relación a los contenedores de menor capacidad. De acuerdo con Bomfim et al. (2009), esta superioridad puede estar relacionada al mayor volumen de sustrato de los recipientes, y en consecuencia, a la mayor disponibilidad de nutrientes y agua. Díaz (2009) indica que los mayores tamaños de contenedores producen los mayores crecimientos en diámetro y altura en algarrobo blanco. Resultados similares fueron obtenidos por Cunha et al. (2005) en Tabebuia impetiginosa (Mart. ex DC.) Standley, Brachtvogel y Malavasi (2010) en Pelthophorum dubium (Spreng.) Taub. y Quiroz et al. (2014) en Acacia dealbata Link., concluyendo que los mayores crecimientos se producen en los recipientes de mayores dimensiones, similar a lo encontrado en este estudio.

Las diferencias de crecimiento entre los dos viveros probablemente tiene relación con el tipo de riego y con las características climáticas de cada localidad, principalmente la temperatura, ya que los algarrobos (Prosopis spp.) en general tienen requerimientos térmicos altos (Vega Rivero et al. 2011). En Concordia, el riego aplicado por aspersión, realizado tres veces al día y con 45 minutos de duración, provocó algunos problemas de encharcamiento en el sustrato de corteza de pino compostada y tierra, dada la mayor capacidad de retención de agua de este sustrato. Por ello se presume que esa fue la causa por la que los plantines bajo estas condiciones presentaron crecimientos sensiblemente menores que en el sustrato de corteza de pino compostada, perlita y vermiculita, que presenta mejor drenaje. En 
Fernández, con riego manual tres veces al día hasta saturación, contrariamente a Concordia, los plantines de ambos sustratos, a igual tamaño de contenedor, presentaron crecimientos en diámetros y alturas similares durante el ciclo productivo, siendo levemente superiores en el sustrato de corteza de pino compostada y tierra, presumiblemente por una mayor eficiencia en la aplicación del riego (menor percolación) y una mayor retención del agua en este sustrato que en la mezcla a base de perlita y vermiculita en el cual el agua se evaporó más rápido.

El exceso de agua en la rizósfera determina una menor disponibilidad de oxígeno, que afecta el crecimiento de las raíces y la absorción de nutrientes (Mascarini et al. 2012). Esta es la causa fundamental de que el suelo, como único componente de sustrato, no deba ser utilizado para el cultivo en contenedor (Acosta Duran et al. 2008), además de dificultar la extracción del plantín del contenedor, de ahí la conveniencia de emplear sustratos con una elevada porosidad, con un mínimo de 50-55\% de poros de aire (Mathers et al. 2007).

Las mayores temperaturas (media, máxima y mínima media) durante el periodo de producción se registraron en la ciudad de Fernández, con diferencias de hasta $4{ }^{\circ} \mathrm{C}$ en comparación con Concordia. En las plantas, un incremento de la temperatura generalmente provoca un aumento del crecimiento, hasta un punto óptimo a partir del cual este se ve afectado (Lambers et al. 1998, Mathers et al. 2007). En plantines, esto puede traducirse en mayores crecimientos o en una mayor elongación de las raíces (Lambers et al. 1998).

Los problemas de deformaciones radiculares (enrulamiento y/o estrangulamiento), se deben al diseño del contenedor o a la permanencia excesiva del plantín dentro del mismo. Si el recipiente es demasiado estrecho (diámetro pequeño), las raíces no tienden a crecer lateralmente, efecto más marcado si los contenedores son profundos (Mathers et al. 2007). Si bien en los contenedores grandes el problema del enrulamiento es menor, estos ocupan más espacio en vivero y prolongan el tiempo necesario para producir un cepellón firme, lo que incrementa los costos de producción, dificultando el almacenamiento, transporte y plantación (Luna et al. 2012).

El patrón de calidad de los plantines varía con la especie que sea considerada, y generalmente se encuentra basada en aspectos fenotípicos que se denominan parámetros morfológicos (altura, diámetro, estado sanitario, entre otros). Para Prosopis sp., Díaz (2009) y Salto et al. (2013) consideran que el plantín con características morfológicas deseables debe presentar una altura entre $20-25 \mathrm{~cm}$ y un diámetro a la altura del cuello entre 2,5-3 $\mathrm{mm}$, además de verse vigorosas y con buen estado sanitario. Para lograr un plantín con las características mencionadas, y fundamentalmente la obtención de un cepellón firme, en Concordia el tiempo de permanencia en el vivero estuvo comprendido entre 80-100 días para los contenedores de dimensiones pequeñas y medianas y entre 120-140 días para los de mayores dimensiones (R250 y R270). En el caso de
Fernández, los plantines producidos en los contenedores de menores dimensiones estuvieron listos entre los 70-80 días y los de mayores dimensiones entre los 90-100 días.

Estos aspectos resaltan la importancia de encontrar un equilibrio entre una producción con menores costos y al mismo tiempo obtener plantines de buena calidad.

\section{CONCLUSIONES}

Las propiedades físicas son dependientes de las características del contenedor y del sustrato, registrándose los mayores valores de porosidad total y porosidad de aireación en el sustrato de corteza de pino compostada, perlita y vermiculita, mientras que la de retención de agua fue mayor en el sustrato de corteza de pino compostada y tierra. Para ambos sustratos, los contenedores más profundos y de mayores dimensiones provocaron mayor porosidad de aireación y menor retención de agua.

Los crecimientos en diámetro al cuello y altura total en Concordia son mayores en el sustrato de corteza de pino compostada, perlita y vermiculita y en contenedores de mayor capacidad (R250 y R270). En Fernández, contenedores de mayores dimensiones (R270 y R250) permiten obtener mayores crecimientos, sin diferencias en cuanto al sustrato empleado al final de ciclo. En ambos sustratos y sitios, los menores crecimientos en diámetro al cuello y altura total se obtuvieron en los contenedores R110 y R100.

Existe una tendencia de mayor crecimiento de los plantines con el aumento del volumen y del diámetro del contenedor, siendo menor la influencia de la profundidad de los mismos.

El tiempo de formación del cepellón es directamente proporcional al tamaño del contenedor. La formación del cepellón es favorecida en el sustrato de corteza de pino compostada, perlita y vermiculita presentando mayor número de raicillas, mayor firmeza y sin disgregación.

Los diferentes contenedores y sustratos evaluados en ambas condiciones permiten ampliar las alternativas del productor de plantines de algarrobo blanco, hasta recientemente limitado a la producción en bolsitas plásticas y tierra como sustrato. La decisión de adoptar una u otra combinación de sustrato y contenedor va a depender del tipo de plantín que se quiera producir, del sustrato disponible y del manejo adoptado por cada vivero.

\section{AGRADECIMIENTOS}

A los proyectos de INTA ERIOS1263305 y PNFOR1104073 por el financiamiento de esta investigación. A los auxiliares de campo que colaboraron en la conducción, mantenimiento y mediciones de los ensayos.

\section{REFERENCIAS}

Acosta Duran CM, CS Gallardo, AN Kämpf, FC Bezerra. 2008. Materiales regionales utilizados en Latinoamérica para la 
preparación de sustratos. Investigación Agropecuaria 5(2): 93-106.

Bomfim AA, AB de Novaes, AR São Jose, FA Grisi. 2009. Avaliação morfológica de mudas de madeira-nova (Pterogyne nitens Tull.) produzidas em tubetes e sacos plásticos e de seu desempenho no campo. Floresta 39(1): 33-40.

Brachtvogel EL, UC Malavasi. 2010. Volume do recipiente, adubação e sua forma de mistura ao substrato no crescimento inicial de Peltophorum dubium (Sprengel) Taubert em viveiro. Árvore 34(2): 223-232.

Correa Londoño G. 2004. Análisis de medidas repetidas. Medellín, Colombia. Facultad de Ciencias Agropecuarias. Universidad Nacional de Colombia. $41 \mathrm{p}$.

Cunha AO, LA Andrade, RLA Bruno, JAL da Silva, VC Souza. 2005. Efeitos de substratos e das dimensões dos recipientes na qualidade das mudas de Tabebuia impetiginosa (Mart. ex D.C.) Standl. Árvore 29(4): 507-516.

Díaz VF. 2009. Comportamiento en Vivero de Prosopis alba Griseb. según sustratos, tipos de envases y dosis de fertilizante. Formosa, Argentina. Tesina de grado. Universidad Nacional de Formosa. 90 p.

García MA, DE Díaz, M Alorda, C Gallardo, O Valenzuela. 2005. Características de los sustratos utilizados por los viveros forestales. IDIA XXI(8): 57-59.

Gomes JM, L Couto, H Garcia Leite, A Xavier, SL Ribeiro Garcia. 2003. Crescimento de mudas de Eucalyptus grandis em diferentes tamanhos de tubetes e fertilização N-P-K. Árvore 27(2): 113-127.

Indrasumunar A, PM Gresshoff. 2013. Vermiculite's strong buffer capacity renders it unsuitable for studies of acidity on soybean (Glycine max L.) nodulation and growth. BMC Research Notes 2013 6: 465. DOI: 10.1186/1756-0500-6-465

Lambers H, FS Chapin, TL Pons. 1998. Plant physiological ecology. Berlín, Alemania. Springer. 540 p.

Landis TD. 1990. Contenedores y medios de crecimiento. In Landis TD, RW Tinus, SE McDonald, JP Barnett eds. Manual de viveros para la producción de especies forestales en contenedor. Agric. Handbook. Washington DC, USA. Department of Agriculture, Forest Service. p. 1-89.

Lemaire F, A Dartigues, LM Rivière, S Charpentier, P Morel. 2005. Cultivos en macetas y contenedores. Principios agronómicos y aplicaciones. Madrid, España. Ediciones Mundi Prensa. 210 p.

López C, A Maldonado, V Salim. 2001. Variación genética de progenies de Prosopis alba. Investigación Agraria: Siste- mas y Recursos Forestales 10(1): 59-68.

Luna T, TD Landis, RK Dumroese. 2012. Contenedores: aspectos técnicos, biológicos y económicos. In Contardi L, $\mathrm{H}$ Gonda eds. Producción de plantas en viveros forestales. Buenos Aires, Argentina. p. 78-85.

Masaguer A, MC Lopez Cuadrado. 2006. Sustratos para viveros. Viveros II Extra 2006. Horticultura Internacional: 44-51.

Mascarini L, G Lorenzo, H Svartz, S Pesenti, S Amado. 2012. Tamaño del contenedor y tipo de sustrato afectan la eficiencia en el uso del agua en Gerbera jamesonii para flor cortada. Revista Brasileira de Horticultura Ornamental 18(1): 71-77.

Mathers HM, SB Lowe, C Scagel, DK Struve, LT Case. 2007. Abiotic factors influencing root growth of woody nursery plants in containers. HortTechnology 17: 151-162.

Nicoloso FT, RP Fortunato, F Zanchetti, LF Cassol, SM Eisinger. 2000. Recipientes e substratos na produção de mudas de Maytenus ilicifolia e Apuleia leiocarpa. Ciência Rural 30(6): 987-992.

Quiroz I, MP Pincheira, JA Hernandez, M Gonzalez, E Garcia, H Soto. 2014. Efecto del volumen radicular sobre el crecimiento de Acacia dealbata Link. en vivero y en terreno en el secano de la Región del Biobío, Chile. Árvore 38(1): 155-164.

Salifu KF, DF Jacobs. 2006. Characterizing fertility targets and multi-element interactions in nursery culture of Quercus rubra seedlings. Annals of Forest Science 63: 231-237.

Salto CS, MA García, L Harrand. 2013. Influencia de diferentes sustratos y contenedores sobre variables morfológicas de plantines de dos especies de Prosopis. Quebracho 21(1,2): 90-101.

Silveira RLVA, EN Higashi, F Sgarbi, MRA Muniz. 2001. Seja o doutor do seu eucalipto. Arquivo do Agrônomo 12. Informações Agronômicas No 93. Piracicaba, Brasil. Potafos. $32 \mathrm{p}$.

Torres AP, D Camberato, RG López, M Mickelbart. 2010. Medición de $\mathrm{pH}$ y conductividad eléctrica en sustratos. Purdue Extension HO-237-SW. West Lafayette, USA. Purdue University. 6 p.

Vega Rivero C, PA Meglioli, PE Villagra. 2011. Prosopis alpataco Phil. (Fabaceae, Mimosoideae). Kurtziana 36(2): 53-64.

Vence LB, OR Valenzuela, HA Svartz, ME Conti. 2013. Elección del sustrato y manejo del riego utilizando como herramienta las curvas de retención de agua. Ciencia del Suelo 31(2): 153-164. 
